\title{
Quercetin supplementation and its effect on human monocyte gene expression profiles in vivo
}

\author{
Inka Boomgaarden ${ }^{1}$, Sarah Egert ${ }^{2}$, Gerald Rimbach ${ }^{3}$, Siegfried Wolffram ${ }^{4}$, Manfred J. Müller ${ }^{2}$ \\ and Frank Döring ${ }^{1 *}$ \\ ${ }^{1}$ Department of Molecular Prevention, Institute of Human Nutrition and Food Science, Christian-Albrechts University Kiel, \\ 24118 Kiel, Germany \\ ${ }^{2}$ Department of Human Nutrition, Institute of Human Nutrition and Food Science, Christian-Albrechts University Kiel, \\ 24105 Kiel, Germany \\ ${ }^{3}$ Department of Food Science, Institute of Human Nutrition and Food Science, Christian-Albrechts University Kiel, \\ 24118 Kiel, Germany \\ ${ }^{4}$ Institute of Animal Nutrition and Physiology, Christian-Albrechts University Kiel, 24118 Kiel, Germany \\ (Received 27 August 2009 - Revised 25 January 2010 - Accepted 26 January 2010 - First published online 26 April 2010)
}

Quercetin has been described as having a wide range of beneficial effects in humans, ranging from anti-carcinogenic properties to reducing the risk of CVD. Nevertheless, underlying molecular mechanisms have been mostly investigated in vitro. Here, we tested whether a daily supplementation of quercetin leads to reproducible changes in human monocyte gene expression profiles. In study I, quercetin in varying dosages was given to healthy subjects for 2 weeks. RNA from monocytes isolated at the beginning and end of the study from subjects receiving $150 \mathrm{mg}$ quercetin per d was subjected to transcriptome-wide microarray analysis. In study II, a double-blind cross-over study, twenty subjects exhibiting a 'cardiovascular risk phenotype' received $150 \mathrm{mg}$ quercetin or placebo daily for 6 weeks each and served as the verification group. Microarray analysis revealed a number of differentially expressed genes. The most significantly represented functional groups were those of the immune system, nucleic acid metabolism, apoptosis and $O$-glycan biosynthesis. Twenty-four genes were chosen for technical replication and independent verification by quantitative real-time PCR. When comparing placebo and quercetin treatment, four genes showed significantly different expression changes (C1GALT1, O-glycan biosynthesis; GM2A, glycolipid catabolism; HDGF, cell proliferation; SERPINB9, apoptosis). However, these were minimal in respect to magnitude of fold change. In conclusion, although microarray analysis revealed extensive effects of quercetin on gene expression, the employment of a placebo-controlled study design showed no comparable results for twenty-four verification targets. This emphasises the need for stringent designs in nutritional intervention studies with the aim to identify relevant changes in gene expression.

Quercetin: Microarrays: Placebo-controlled studies: Monocytes

Quercetin $\left(3,3^{\prime}, 4^{\prime}, 5,7\right.$-pentahydroxyflavone $)$ is a naturally occurring polyphenol belonging to the flavonoid subclass of flavonols. The main dietary sources of quercetin include tea, red wine, fruits (especially apples) and vegetables (for example, onions), where quercetin is found mainly as $\beta$-glycoside. The daily intake of quercetin has been estimated at $14-18 \mathrm{mg}$ in Western populations ${ }^{(1,2)}$. After ingestion quercetin is subject to hydrolysation and conjugation with glucuronic acid and/or sulfate, with quercetin3 -glucuronide and quercetin- $3^{\prime}$-sulfate constituting the main metabolites in human plasma ${ }^{(3)}$. In recent years, dietary polyphenols in general, and quercetin in particular, have attracted considerable attention regarding potential health benefits. In epidemiological and intervention studies, effects of flavonoid intake on CVD, inflammation and cancer have been described (for reviews, see Arts \& Hollman ${ }^{(4)}$ and Williamson
$\&$ Manach $)^{(5)}$. The effects of quercetin may be attributed in part to its antioxidative capacity, enabling it to act as a potent scavenger of reactive oxygen and nitrogen species, which are believed to play an important role in several diseases, such as cancer, CVD and insulin resistance ${ }^{(6-8)}$. Quercetin has been described as a kinase inhibitor and to affect apoptosis and cell-cycle control ${ }^{(9)}$. The actions of quercetin on the molecular level have been investigated mainly in cell-culture experiments using cancer cell lines treated with supraphysiological concentrations of quercetin aglycone, although there is an increasing body of work investigating quercetin metabolites occurring in vivo ${ }^{(10-12)}$. With the advent of microarray-based technologies, the effects of quercetin on gene expression have been investigated more thoroughly ${ }^{(13-15)}$. Nevertheless, extrapolation of results obtained in cell culture to an in vivo situation,

Abbreviations: FC, fold change; FDR, false discovery rate; GO, Gene Ontology; LDA, low-density array; qRT-PCR, quantitative real-time PCR; Q50, study I 50 mg quercetin supplementation; Q100, study I $100 \mathrm{mg}$ quercetin supplementation; Q150, study I 150 mg quercetin supplementation.

* Corresponding author: Professor Dr Frank Döring, fax +49 431 8805658, email sek@molprev.uni-kiel.de 
despite providing valuable data on principal mechanisms of action, is often limited. The number of studies investigating the effect of quercetin on gene expression in vivo is small and most work has been conducted on laboratory rodents fed a quercetin-enriched diet ${ }^{(13,16,17)}$ with subsequent microarray analysis on specific tissues. Human studies were either conducted ex vivo with quercetin or in vivo using quercetin-rich foods rather than the pure compound ${ }^{(18-20)}$ and addressed the expression of only selected genes. The aims of the present research were to investigate, for the first time, the in vivo effect of quercetin on transcriptomewide gene expression in CD14-positive monocytes with microarray techniques in apparently healthy human subjects. In addition, dose-dependent effects of quercetin on the expression of selected genes were analysed. To verify the present results we employed subjects exhibiting a 'cardiovascular risk phenotype' from a placebo-controlled double-blind trial receiving the same quercetin supplementation as the subjects of the microarray experiment.

\section{Experimental methods}

\section{Subjects and study designs}

Study I. Ten healthy volunteers (six male, four female) were chosen randomly from the three quercetin supplementation groups of a nutritional trial at Kiel University as described previously $^{(21)}$. In brief, the double-blind parallel study consisted of a 2-week wash-out period followed by a 2-week supplementation period, where subjects were randomly assigned to one of three supplementation groups (study I). Supplementation groups received either $50 \mathrm{mg}$ (Q50), $100 \mathrm{mg}$ (Q100) or $150 \mathrm{mg}$ (Q150) quercetin (aglycone) per $\mathrm{d}$ in the form of six hard gelatin capsules, two capsules with each principal meal. In addition to four subjects from group Q150 selected for microarray analysis, three subjects from group Q50 and two subjects from group Q100 were chosen for this study.

Study II. Forty-two volunteers (twelve male, twelve female) were chosen from a group of ninety-three subjects taking part in a quercetin-supplementation study at Kiel University (study II) ${ }^{(22)}$. Subjects in study II were chosen on the basis of exhibiting a 'cardiovascular risk phenotype' with elevated BMI $\left(25-35 \mathrm{~kg} / \mathrm{m}^{2}\right)$, central obesity (waist circumference $\geq 94 \mathrm{~cm}$ for men and $\geq 80 \mathrm{~cm}$ for women), serum concentrations of TAG $\geq 1.7 \mathrm{mmol} / \mathrm{l}$ and/or C-reactive protein $\geq 2.0 \mathrm{mg} / \mathrm{l}$. Briefly, in a double-blind, placebocontrolled, cross-over design the subjects received either $150 \mathrm{mg}$ quercetin (aglycone) (see above) or placebo capsules. The supplementation period lasted 6 weeks and after a washout period of 5 weeks' treatment was swapped between groups. Twenty subjects successfully completed the study (eleven male, nine female).

Both studies were approved by the ethical committee of the Medical Faculty of the University of Kiel and subjects gave their written consent after detailed explanation. The study protocols were in accordance with the Helsinki Declaration of 1975, as revised 1983. Laboratory measurements including serum lipid concentrations, plasma TNF- $\alpha$, C-reactive protein and quercetin concentrations (including metabolites) have been described previously ${ }^{(21)}$.

\section{Monocyte isolation and RNA extraction}

After an overnight fasting period $27 \mathrm{ml}$ venous blood were drawn into three $9 \mathrm{ml} \mathrm{K}$-EDTA monovettes at the beginning and end of each intervention period. The monovettes were stored at $4^{\circ} \mathrm{C}$ until isolation of the monocytes. CD14-positive monocytes were isolated by density centrifugation with LymphoPrep $^{\text {TM }}$ (Progen, Wieblingen, Germany) and successive positive selection with magnetic beads (Miltenyi Biotec $\mathrm{GmbH}$, Bergisch Gladbach, Germany) as described previously $^{(23)}$. CD14-positive monocytes were counted with a haemocytometer and stored at $-80^{\circ} \mathrm{C}$ until all the samples from one study were completed. Total RNA was extracted with the RNeasy Mini Kit, including an on-column DNase digestion step according to the manufacturer's instructions (Qiagen, Hilden, Germany). RNA concentration was determined spectrophotometrically and RNA integrity was measured with a 2100 Bioanalyser using the RNA 6000 Nano Kit (Agilent Technologies, Santa Clara, CA, USA).

\section{Microarray analysis}

Samples from three subjects from supplementation group Q150 of study I were chosen for microarray analysis based on quality and quantity of isolated RNA. RNA integrity, given as RNA integrity number, ranged between 7.6 and 8.9 for the six samples. Biotin-labelled cRNA for hybridisation was synthesised from $2 \mu \mathrm{g}$ total RNA with the MessageAmp $^{\text {TM }}$ II-Biotin Enhanced Kit (Ambion/Applied Biosystems, Darmstadt, Germany) including poly-A RNA controls (GeneChip ${ }^{\circledR}$ Eukaryotic Poly-A RNA Control Kit) according to the manufacturer's instructions. Six Human Genome U133 + 2.0 GeneChip ${ }^{\circledR}$ microarrays were hybridised for $16 \mathrm{~h}$ at $45^{\circ} \mathrm{C}$ with $15 \mu \mathrm{g}$ fragmented, biotin-labelled cRNA each, including hybridisation controls (GeneChip ${ }^{\circledR}$ Eukaryotic Hybridization Control Kit), using a Hybridization Oven 640 and following the manufacturer's instructions (GeneChip ${ }^{\circledR}$ Expression Analysis Technical Manual, P/N 702232 Rev. 2). Microarrays were subsequently washed and stained using a Fluidics Station 450 and the FS450_0004 script. Solutions used for hybridisation, washing and staining were taken from the GeneChip ${ }^{\circledR}$ Hybridization, Wash and Stain Kit. After hybridisation and washing, microarrays were scanned with a GeneChip ${ }^{\circledR}$ Scanner 3000 using the GCOS software. Unless otherwise stated, all kits and equipment were purchased from Affymetrix (High Wycombe, Bucks, UK). Fluorescence data were obtained in CEL-file format. Quality control and normalisation of CEL data based on the Affymetrix chip definition file were performed with $\mathrm{R}$ software (version 2.7.1) and BioConductor (version 2.0.1) with the package 'simpleaffy' (version 2.16.0-1) as provided by the MADMAX database (http://madmax.bioinformatics.nl). Data were normalised with the GC-RMA algorithm and background correction based on empirical Bayes estimate (GC-RMA slow, 'gcrma' package version 2.12.1). In addition, MAS5.0 normalisation was conducted with Affymetrix Expression Console software (version 1.1) to obtain present and absent calls (P/A) for each probe set. To identify differentially expressed probe sets these were first filtered by P/A calls. Only probe sets with a P-call for all six microarrays were included for further analysis. Only probe sets with the same direction of 
expression change in all three subjects were retained (measured as ratio of normalised fluorescence after quercetin supplementation to fluorescence at baseline). To identify statistical significance in gene expression changes, a two-sided, paired-sample Student's $t$ test was calculated for normalised fluorescence data. Probe sets were considered to be differentially expressed if the mean fold change (FC) was at least 1.5 -fold (without $P$ value cut-off condition) or 1.3 -fold with $P \leq 0 \cdot 01$. For the resulting list of probe sets, the corresponding Entrez gene identifiers were obtained with NetAffx (annotation version current in October 2008; http://www.affymetrix. com/analysis/index.affx). The resulting list was manually reviewed, removing probe sets with no corresponding Entrez gene identifier, probe sets with conflicting results for the same transcript, and multiple entries, retaining only data from those with the highest mean FC or lowest $P$ value per gene. Functional annotation enrichment analysis was conducted with DAVID (Database for Annotation, Visualization and Integrated Discovery) Bioinformatics Resources (http:// david.abcc.ncifcrf.gov) ${ }^{(24)}$ using the list of differentially expressed transcripts and $\mathrm{HG} \mathrm{U} 133+2.0$ background, as provided by DAVID. Gene ontology (GO) annotations of biological processes (at level 4) with a false discovery rate (FDR) below $10 \%(P<0.005$, Fisher exact test $)$ and pathways from KEGG (Kyoto Encyclopedia of Genes and Genomes) with an FDR below $15 \%(P<0.05)$ were considered significant. All microarray datasets and sample information have been submitted in Minimal Information About Microarray Experiments-compliant format ${ }^{(25)}$ to the National Center for Biotechnology Information Gene Expression Omnibus repository (http://www.ncbi.nlm.nih.gov/geo/) under accession number GSE13899.

\section{Quantitative real-time PCR}

For technical and biological replication of microarray results, twenty-two genes were chosen based on magnitude of FC, significance of expression change after quercetin supplementation and biological relevance (for example, involved in inflammatory process, redox homeostasis, etc) or combinations thereof. Replication experiments were conducted with quantitative real-time PCR (qRT-PCR), using the TaqMan ${ }^{\circledR}$ System (Applied Biosystems, Darmstadt, Germany) and custom-manufactured low-density arrays (LDA) with glyceraldehyde-3-phosphate dehydrogenase $(G A P D H)$ and TATA box binding protein $(T B P)$ serving as endogenous controls. The TaqMan ${ }^{\circledR}$ Assays and corresponding order numbers are given in Supplemental Table S2. The LDA used in this experiment consisted of 384-well micro fluidic cards preloaded in duplicate with the chosen TaqMan ${ }^{\circledR}$ Assays. Total RNA $(1 \mu \mathrm{g})$ from participants in study I (three participants in group Q50, two participants in group Q100 and three participants in group Q150) and from study II (twenty participants, four samples each) was reverse transcribed with iScript (Bio-Rad, Munich, Germany) according to the manufacturer's instructions. cDNA $(200 \mathrm{ng})$ and $50 \mu \mathrm{l}$ of TaqMan ${ }^{\circledR}$ Universal Mastermix (Applied Biosystems, Darmstadt, Germany) in a total volume of $100 \mu \mathrm{l}$ were loaded per sample and cavity, and after centrifugation fluorescence was measured with the ABI 7900 HT Fast Real-time PCR System (Applied Biosystems). Multiple samples from one subject were always loaded onto the same LDA to avoid introducing technical, in addition to intra-individual, variation. Expression values of one of the genes measured with LDA and two additional genes were measured with conventional TaqMan ${ }^{\circledR}$ qRT-PCR in the ninety-six-well format. Each reaction contained $20 \mathrm{ng}$ cDNA, $1 \mu \mathrm{l}$ TaqMan ${ }^{\circledR}$ Assay and $10 \mu$ l Universal TaqMan ${ }^{\circledR}$ Mastermix in a total volume of $20 \mu \mathrm{l}$ and all measurements were performed in triplicate with an ABI 7300 Real-time PCR System (Applied Biosystems). Assays were tested for PCR efficiency $(100 \pm 5 \%)$ and applicability of the comparative cycle threshold $\left(\mathrm{C}_{\mathrm{T}}\right)$ method (slope of semi-log regression line $\Delta \mathrm{C}_{\mathrm{T}} v . \log$ input $\left.<0 \cdot 1\right)$ with standard curves performed in triplicate with cDNA from the human acute monocytic leukaemia cell line THP-1 ${ }^{(26,27)}$. All FC in gene expression were calculated with the comparative $C_{T} \operatorname{method}^{(28)}$ using glyceraldehyde-3-phosphate dehydrogenase as the endogenous control. Glyceraldehyde-3-phosphate dehydrogenase was chosen based on lower variance in expression over all samples, as measured by LDA, compared with TATA box binding protein (data not shown).

\section{Statistical analysis}

Statistics were calculated with SPSS 11.0 software (SPSS GmbH Software, Munich, Germany) and Microsoft Office Excel 2003 (Microsoft Corp., Redmond, WA, USA) if not stated otherwise. Characteristics of the study subjects were tested for normal distribution with the Shapiro-Wilk test. Normally distributed variables and data from more than four observations are given as arithmetic mean values and standard deviations. Not-normally distributed variables are given as medians and 25th and 75th percentiles using Tukey's hinges. Dependence of gene expression values on quercetin dose given in study I was calculated by linear regression and expressed as coefficient of correlation $R^{2}$.

\section{Results}

\section{Study populations}

To identify effects of quercetin supplementation on gene expression in vivo we analysed CD14-positive human monocytes from the participants of two studies (study designs illustrated in Fig. 1). Participants of study I (dose-dependence study) received 50, 100 or $150 \mathrm{mg}$ quercetin per $\mathrm{d}$ for 2 weeks and compliance with treatment was $97 \%$ (determined by counting returned capsules). Baseline characteristics of the subjects, including serum lipid profiles and TNF- $\alpha$ as a marker of inflammation, are given in Table 1. Plasma quercetin concentrations increased significantly and dose-dependently within the 2-week supplementation period (before and after supplementation: Q50, 58.8 (SD 5.3) v. 156.1 (SD 85.9) $\mathrm{nmol} / \mathrm{l}$; Q100, 77.0 and 46.8 v. 167.0 and $172.2 \mathrm{nmol} / \mathrm{l}$; Q150, 87.6 (SD 51.1) v. 401.8 (SD 179.2) nmol/1) as shown in Table 1 and discussed by Egert et al. ${ }^{(21)}$.

Participants of study II (cross-over study) received $150 \mathrm{mg}$ quercetin per $\mathrm{d}$ or placebo in a cross-over design for 6 weeks each. Compliance with treatment was $98 \%$. Baseline characteristics of the subjects are given in Table 2. Median plasma quercetin concentrations were significantly elevated after quercetin supplementation compared with placebo 
(a)
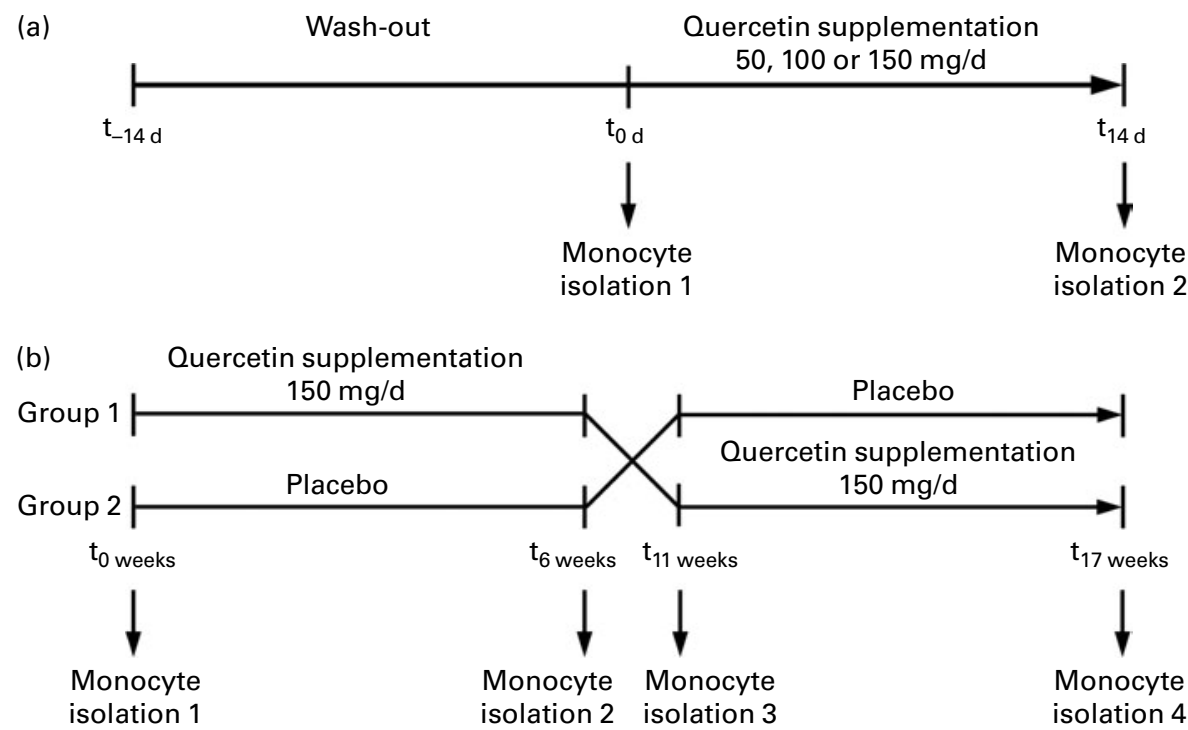

Fig. 1. Diagram of study designs employed in this investigation. (a) Study I (dose-dependence), with $n 9$ (50 mg quercetin/d, $n 3 ; 100 \mathrm{mg}$ quercetin/d, $n 2 ; 150 \mathrm{mg}$ quercetin/d, $n$ 4). (b) Study II (double-blind, placebo-controlled cross-over), with $n 20$ (group 1, $n$ 11; group 2, $n$ 9).

treatment, independent of the subjects receiving quercetin in phase 1 or 2 of the cross-over study (before and after quercetin supplementation: $61 \cdot 1 \quad(25$ th -75 th percentile $38 \cdot 9-70 \cdot 1)$ v. $279.9(25$ th -75 th percentile $196.7-374.6) \mathrm{nmol} / \mathrm{l}$ and $62 \cdot 2$ (25th-75th percentile 52.9-64.9) v. 239.5 (25th-75th percentile 227.0-270.4) nmol/l).

\section{Microarray analysis of differentially expressed} genes after quercetin supplementation

To elucidate molecular mechanisms of quercetin action in vivo we measured transcriptome profiles of CD14-positive monocytes from three subjects before and after a 2-week quercetin supplementation of $150 \mathrm{mg} / \mathrm{d}$ (study I). The workflow from scanning of microarrays to the list of differentially expressed genes is depicted in Supplemental Fig. S1. Based on the selection criteria described in Experimental methods, analysis of the microarray experiments yielded a list of 788 unique genes identifiable by Entrez gene identifiers. The expression of 503 genes was up-regulated and 285 genes down-regulated after quercetin supplementation with a magnitude of FC ranging from $1 \cdot 3$ to $26 \cdot 3$ and $-1 \cdot 3$ to $-3 \cdot 6$, respectively.

To identify biological processes affected by quercetin supplementation, the list of differentially expressed genes was subjected to gene-annotation enrichment analysis using DAVID (Database for Annotation, Visualization and Integrated Discovery) bioinformatics resources and the GO vocabulary. This approach mined results from the microarray experiment for over-representation of genes associated with specific terms in contrast to what would be expected by

Table 1. Characteristics of subjects in study I (dose-dependence)*

(Mean values and standard deviations)

\begin{tabular}{|c|c|c|c|c|c|c|}
\hline \multirow[t]{2}{*}{ Quercetin dose... } & \multicolumn{2}{|c|}{$50 \mathrm{mg} / \mathrm{d}$} & \multicolumn{2}{|c|}{$100 \mathrm{mg} / \mathrm{d}$} & \multicolumn{2}{|c|}{$150 \mathrm{mg} / \mathrm{d}$} \\
\hline & Mean & SD & Subject 1 & Subject 2 & Mean & SD \\
\hline Subjects $(n)$ & \multicolumn{2}{|c|}{3} & \multicolumn{2}{|c|}{2} & \multicolumn{2}{|c|}{4} \\
\hline \multicolumn{7}{|l|}{$\operatorname{Sex}(n)$} \\
\hline Male & \multicolumn{2}{|c|}{2} & \multicolumn{2}{|l|}{1} & \multicolumn{2}{|c|}{2} \\
\hline Female & \multicolumn{2}{|c|}{1} & \multirow{2}{*}{\multicolumn{2}{|c|}{$\begin{array}{r}1 \\
26\end{array}$}} & \multicolumn{2}{|c|}{2} \\
\hline Age (years) & 27 & $4 \cdot 2$ & & & 24 & $2 \cdot 8$ \\
\hline $\mathrm{BMI}\left(\mathrm{kg} / \mathrm{m}^{2}\right)$ & $21 \cdot 9$ & 1.52 & $23 \cdot 6$ & $23 \cdot 0$ & $21 \cdot 1$ & 1.91 \\
\hline Systolic blood pressure (mmHg) & 127 & 12 & 120 & 120 & 122 & 5 \\
\hline Diastolic blood pressure $(\mathrm{mmHg})$ & 77 & 3 & 70 & 75 & 73 & 5 \\
\hline Serum total cholesterol (mmol/l) & $4 \cdot 24$ & 0.23 & 3.96 & $4 \cdot 01$ & $4 \cdot 13$ & $1 \cdot 33$ \\
\hline Serum LDL-cholesterol (mmol/l) & $2 \cdot 28$ & 0.08 & 2.43 & 1.96 & $1 \cdot 81$ & 0.61 \\
\hline Serum HDL-cholesterol (mmol/l) & $1 \cdot 39$ & $0 \cdot 28$ & $1 \cdot 27$ & $1 \cdot 81$ & 1.78 & 0.69 \\
\hline Serum TAG (mmol/l) & $1 \cdot 26$ & 0.80 & 0.56 & 0.52 & $1 \cdot 19$ & 0.42 \\
\hline Serum TNF- $\alpha$ (pg/ml) & 3.90 & $1 \cdot 24$ & $1 \cdot 87$ & 2.78 & $2 \cdot 60$ & 0.39 \\
\hline \multicolumn{7}{|l|}{ Plasma quercetin (nmol/l) } \\
\hline Pre & $58 \cdot 8$ & $5 \cdot 3$ & $77 \cdot 0$ & $46 \cdot 8$ & $87 \cdot 6$ & $51 \cdot 1$ \\
\hline Post & $156 \cdot 1$ & 85.9 & $167 \cdot 0$ & $172 \cdot 2$ & $401 \cdot 8$ & $179 \cdot 2$ \\
\hline
\end{tabular}

pre, Baseline data; post, endpoint data.

* Serum and plasma measurements were done from fasting blood samples. 
chance. Enrichment analysis identified a number of significantly over-represented biological processes (at GO Biological Process level four, covering 522 input genes) summarised as (a) haematopoiesis and immune system-related, (b) nucleic acid metabolism and (c) apoptosis and a number of broader biological process terms (Table 3 and Supplemental Tables S3, S4 and S5). This categorisation includes a number of genes associated with more than one GO term at the same time. All GO terms were associated with up- as well as down-regulated genes. As stated above, the GO annotation of biological processes does not necessarily include pathways. To identify possible perturbations of biological pathways by quercetin, the differentially expressed genes were compared with the KEGG (Kyoto Encyclopedia of Genes and Genomes) pathway database, which includes pathway maps on metabolism, genetic and environmental information processing, cellular processes and human diseases. Functional enrichment analysis (covering 176 input genes) resulted in three significantly over-represented pathways, namely $O$-glycan biosynthesis (six genes, $P=0.009$, FDR $11 \%$ ), adipocytokine signalling (nine genes, $P=0.011$, FDR $14 \%$ ) and pancreatic cancer (nine genes, $P=0.012$, FDR $15 \%$ ), as shown in Table 4. The pathways of non-small cell lung cancer (hsa05223) and apoptosis (hsa04210) showed $P$ values $<0.05$ but an FDR less than 26 and $29 \%$, respectively (data not shown).

\section{Selection of target genes for further verification}

In order to further evaluate the microarray data, twenty-four differentially expressed genes after quercetin supplementation were selected for verification (Table 5). Selection criteria were based on magnitude of FC, significance of FC (assessed by paired Student's $t$ test), biological relevance according to described actions of quercetin (for example, cellular redox status, inflammatory processes, angiogenesis, etc) and the quality of the corresponding probe set sequence (for example, probe sets interrogating single or multiple transcripts; current annotation shows location outside of coding sequence as inspected with UCSC Genome Browser and March 2006 assembly of the Human Genome) or combinations thereof These included haematopoiesis and immune system-related genes (nuclear factor of $\kappa$ light polypeptide gene enhancer in B-cells 2 (NFKB2; FC 1.54), early growth response 1 (EGR1; FC - 2.14) and IL-7 receptor (IL7R; FC - 2.26)), genes of nucleic acid metabolism (structural maintenance of chromosomes 3 (SMC3; FC 6.45), superoxide dismutase 2 (SOD2; FC 3.49) and $I L 7 R$ ) and apoptosis-related genes (SOD2, serpin peptidase inhibitor clade B member 9 (SERPINB9; FC 2.45) and granzyme A (GZMA; FC - 1.89)). Selected genes representing biological pathways were those related to $O$-glycan biosynthesis (glycoprotein-N-acetylgalactosamine 3- $\beta$-galactosyltransferase 1 (C1GALT1; FC 1.7) and ST3 $\beta$-galactoside $\alpha$-2,3-sialyltransferase 1 (ST3GAL1; FC - 1.9)) and adipocytokine signalling and pancreatic cancer (NFKB2 (FC 1.54) and carnitine palmitoyltransferase 1B (CPT1B; FC 2.4)). Additionally selected genes were: GM2 ganglioside activator (GM2A; FC 1.98; glycolipid metabolism), $\mathrm{Hb} \alpha 1$ (HBA1; multiple probe sets FC $2 \cdot 0-4 \cdot 1)$, hepatoma-derived growth factor $(H D G F$; FC -1.5 and $P=0.002$; cell proliferation), opioid receptor sigma 1 (OPRS1; FC $-1 \cdot 3$; possible

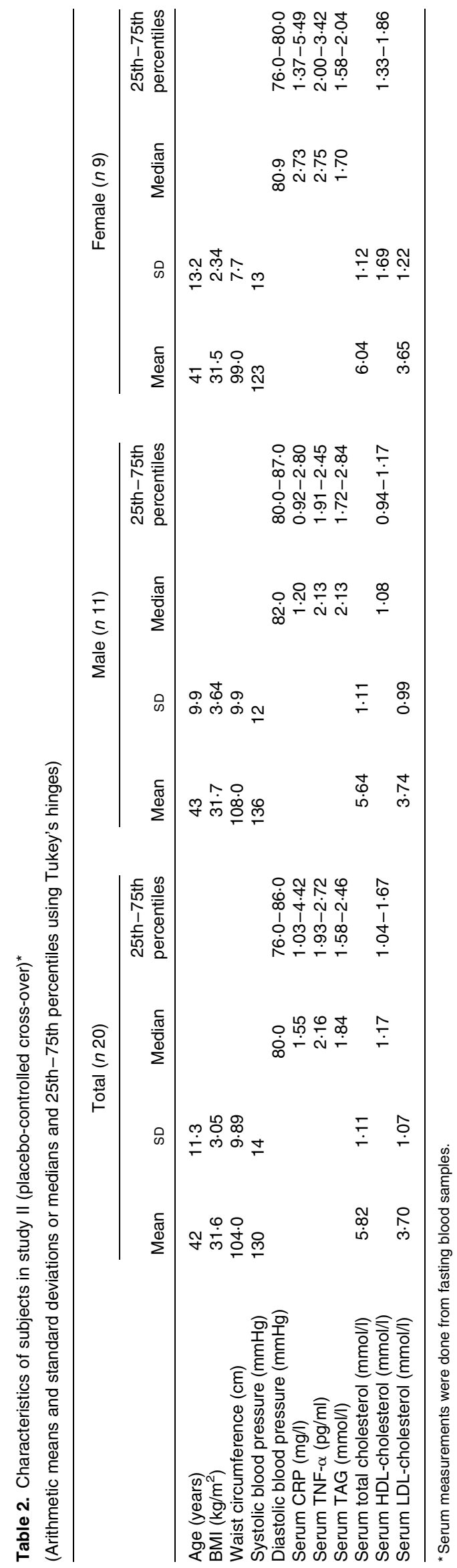


Table 3. DAVID (Database for Annotation, Visualization and Integrated Discovery) gene-annotation enrichment analysis of Gene Ontology (GO) annotated biological processes

\begin{tabular}{|c|c|c|c|c|}
\hline GO ID & GO term & No of input genes & $P^{*}$ & FDR \\
\hline \multicolumn{5}{|c|}{ Haematopoiesis and immune system } \\
\hline 0048534 & Hemopoietic or lymphoid organ development & 28 & $<0.001$ & $<0.001$ \\
\hline 0002520 & Immune system development & 28 & $<0.001$ & 0.002 \\
\hline 0030097 & Hemopoiesis & 26 & $<0.001$ & 0.002 \\
\hline 0030099 & Myeloid cell differentiation & 13 & 0.001 & 1.1 \\
\hline 0002474 & $\begin{array}{l}\text { Antigen processing and presentation of peptide } \\
\text { antigen via MHC class I }\end{array}$ & 7 & 0.001 & $1 \cdot 0$ \\
\hline \multicolumn{5}{|c|}{ Nucleic acid metabolism } \\
\hline 0006259 & DNA metabolic process & 51 & 0.001 & 2.4 \\
\hline 0016070 & RNA metabolic process & 151 & 0.002 & 3.6 \\
\hline 0000375 & RNA splicing, via transesterification reactions & 10 & 0.004 & $5 \cdot 8$ \\
\hline 0000398 & Nuclear mRNA splicing, via spliceosome & 10 & 0.004 & $5 \cdot 8$ \\
\hline \multicolumn{5}{|c|}{ Apoptosis-related } \\
\hline 0012501 & Programmed cell death & 52 & 0.003 & 4.8 \\
\hline 0043068 & Positive regulation of programmed cell death & 22 & 0.005 & $8 \cdot 1$ \\
\hline \multicolumn{5}{|l|}{ Other } \\
\hline 0043412 & Biopolymer modification & 110 & $<0.001$ & 0.1 \\
\hline 0006796 & Phosphate metabolic process & 65 & $<0.001$ & 0.3 \\
\hline 0044267 & Cellular protein metabolic process & 170 & $<0.001$ & 0.7 \\
\hline
\end{tabular}

FDR, false discovery rate; MHC, major histocompatibility complex.

* Modified Fisher exact $P$ value; background $\mathrm{HG} \mathrm{U133+2 \cdot 0;} \mathrm{GO} \mathrm{Biological} \mathrm{Process} \mathrm{level} 4$

Table 4. Significantly over-represented Kyoto Encyclopedia of Genes and Genomes (KEGG) pathways and corresponding genes

\begin{tabular}{|c|c|c|c|c|}
\hline \multirow[b]{2}{*}{ Entrez ID } & \multicolumn{2}{|r|}{ KEGG pathway ID } & \multicolumn{2}{|l|}{ FDR (\%) } \\
\hline & Gene symbol & Gene name & Mean fold change & $P$ \\
\hline O-glycan biosynthesis (hsa00512) & & & 11 & 0.009 \\
\hline 2591 & GALNT3 & $\begin{array}{l}\text { UDP-N-acetyl-alpha-D-galactosamine: polypeptide } \\
\mathrm{N} \text {-acetylgalactosaminyltransferase } 3\end{array}$ & $1 \cdot 8$ & 0.39 \\
\hline 2650 & GCNT1 & $\begin{array}{l}\text { Glucosaminyl (N-acetyl) transferase } 1 \text {, core } 2 \\
\text { (beta-1,6-N-acetylglucosaminyltransferase) }\end{array}$ & $1 \cdot 8$ & 0.40 \\
\hline 56913 & $C 1 G A L T 1^{*}$ & $\begin{array}{l}\text { Core } 1 \text { synthase, glycoprotein- } \mathrm{N} \text {-acetylgalactosamine } \\
\text { 3-beta-galactosyltransferase, } 1\end{array}$ & $1 \cdot 7$ & 0.29 \\
\hline 29071 & C1GALT1C1 & C1GALT1-specific chaperone 1 & 1.5 & 0.31 \\
\hline 8473 & OGT & O-linked $\mathrm{N}$-acetylglucosamine (GlcNAc) transferase & 1.5 & 0.23 \\
\hline 6482 & $S T 3 G A L 1^{*}$ & ST3 beta-galactoside alpha-2,3-sialyltransferase 1 & -1.9 & 0.05 \\
\hline Adipocytokine signalling (hsa04920) & & & 14 & 0.011 \\
\hline 1375 & $C P T 1 B^{*}$ & Carnitine palmitoyltransferase 1B (muscle) & $2 \cdot 4$ & $0 \cdot 10$ \\
\hline 9021 & socs3 & Suppressor of cytokine signalling 3 & $2 \cdot 2$ & 0.21 \\
\hline 2180 & ACSL1 & Acyl-CoA synthetase long-chain family member 1 & $2 \cdot 1$ & 0.36 \\
\hline 3717 & JAK2 & Janus kinase 2 & $2 \cdot 1$ & 0.25 \\
\hline 3716 & $J A K 1$ & Janus kinase 1 & $2 \cdot 0$ & 0.09 \\
\hline 6774 & STAT3 & $\begin{array}{l}\text { Signal transducer and activator of transcription } 3 \\
\text { (acute-phase response factor) }\end{array}$ & $1 \cdot 7$ & 0.39 \\
\hline 2181 & ACSL3 & Acyl-CoA synthetase long-chain family member 3 & 1.6 & 0.32 \\
\hline 51422 & PRKAG2 & $\begin{array}{l}\text { Protein kinase, AMP-activated, gamma } \\
2 \text { non-catalytic subunit }\end{array}$ & $1 \cdot 6$ & 0.34 \\
\hline 4791 & $N F K B 2^{*}$ & $\begin{array}{l}\text { Nuclear factor of kappa light polypeptide gene } \\
\text { enhancer in B-cells } 2 \text { (p49/p100) }\end{array}$ & 1.5 & 0.001 \\
\hline Pancreatic cancer (hsa05212) & & & 15 & 0.012 \\
\hline 6772 & STAT1 & Signal transducer and activator of transcription 1 & $2 \cdot 9$ & 0.39 \\
\hline 3716 & $J A K 1$ & Janus kinase 1 & $2 \cdot 0$ & 0.09 \\
\hline 5894 & RAF1 & v-raf-1 murine leukaemia viral oncogene homologue 1 & $1 \cdot 8$ & 0.13 \\
\hline 6774 & STAT3 & Signal transducer and activator of transcription 3 & $1 \cdot 7$ & 0.39 \\
\hline 5228 & $P G F$ & Placental growth factor & 1.6 & 0.05 \\
\hline 4791 & $N F K B 2^{*}$ & $\begin{array}{l}\text { Nuclear factor of kappa light polypeptide gene enhancer } \\
\text { in B-cells } 2 \text { (p49/p100) }\end{array}$ & 1.5 & 0.001 \\
\hline 91 & $A C V R 1 B$ & Activin A receptor, type IB & $-1 \cdot 3$ & 0.007 \\
\hline 1021 & CDK6 & Cyclin-dependent kinase 6 & -1.5 & 0.26 \\
\hline 1019 & CDK4 & Cyclin-dependent kinase 4 & -1.6 & $0 \cdot 10$ \\
\hline
\end{tabular}

FDR, false discovery rate; Entrez ID, Entrez gene identifier.

* Genes which were also measured by quantitative real-time PCR. 
Table 5. Expression changes of selected transcripts measured by microarray (study I) and low-density array (LDA) quantitative real-time PCR (study II)

\begin{tabular}{|c|c|c|c|c|c|c|c|c|}
\hline \multirow[b]{3}{*}{ Entrez ID } & \multirow[b]{3}{*}{ Gene symbol } & \multirow[b]{3}{*}{ Gene name } & \multicolumn{5}{|c|}{ Mean fold change* } & \multirow[b]{3}{*}{$P$} \\
\hline & & & \multirow[b]{2}{*}{ Microarray } & \multicolumn{2}{|c|}{ Placebo LDA } & \multicolumn{2}{|c|}{ Quercetin LDA } & \\
\hline & & & & Mean & SD & Mean & SD & \\
\hline $56913 † \ddagger$ & C1GALT1 & $\begin{array}{l}\text { Core } 1 \text { synthase, glycoprotein- } \mathrm{N} \text {-acetylgalactosamine } \\
\text { 3-beta-galactosyltransferase, } 1\end{array}$ & $1 \cdot 7$ & $1 \cdot 23$ & 0.42 & 1.02 & 0.22 & 0.034 \\
\hline $1375 \S$ & CPT1B & Carnitine palmitoyltransferase 1B (muscle) & 2.39 & $1 \cdot 15$ & 0.35 & 1.09 & 0.30 & 0.561 \\
\hline $1958 \|$ & EGR1 & Early growth response 1 & -1.85 & 1.43 & $2 \cdot 29$ & 1.34 & $1 \cdot 17$ & 0.816 \\
\hline 2760 & GM2A & GM2 ganglioside activator & 1.98 & 1.08 & 0.32 & -1.08 & 0.16 & 0.043 \\
\hline 2878 & GPX3 & Glutathione peroxidase 3 (plasma) & $-1 \cdot 60$ & -1.05 & 0.30 & $-1 \cdot 11$ & 0.43 & 0.735 \\
\hline 30019 & GZMA & $\begin{array}{l}\text { Granzyme A (granzyme 1, cytotoxic } \\
\text { T-lymphocyte-associated serine esterase 3) }\end{array}$ & $-1 \cdot 89$ & -1.05 & $0 \cdot 80$ & 1.02 & 0.70 & 0.788 \\
\hline 3039 & $H B A 1$ & Haemoglobin, alpha 1 & $2 \cdot 80$ & $2 \cdot 24$ & $3 \cdot 20$ & 1.47 & 1.44 & 0.248 \\
\hline 3068 & HDGF & $\begin{array}{l}\text { Hepatoma-derived growth factor } \\
\text { (high-mobility group protein 1-like) }\end{array}$ & $-1 \cdot 47$ & $-1 \cdot 15$ & $0 \cdot 14$ & -1.05 & 0.16 & 0.049 \\
\hline $3575 \|^{* *}$ & IL7R & Interleukin 7 receptor & $-2 \cdot 26$ & -1.31 & 0.52 & 1.10 & 0.67 & 0.125 \\
\hline 8826 & IQGAP1 & IQ motif containing GTPase activating protein 1 & $26 \cdot 28$ & -1.09 & 0.12 & -1.05 & 0.19 & 0.412 \\
\hline 378938 & MALAT1 & $\begin{array}{l}\text { Metastasis associated lung adenocarcinoma } \\
\text { transcript } 1 \text { (non-protein coding) }\end{array}$ & $4 \cdot 69$ & $1 \cdot 11$ & 0.36 & $1 \cdot 13$ & 0.42 & 0.825 \\
\hline 23269 & $M G A$ & MAX gene associated & -1.97 & 1.03 & 0.21 & -1.09 & 0.17 & 0.062 \\
\hline 11343 & MGLL & Monoglyceride lipase & -1.64 & -1.05 & 0.34 & -1.05 & 0.29 & 0.995 \\
\hline $4791 \|$ & NFKB2 & $\begin{array}{l}\text { Nuclear factor of kappa light polypeptide gene } \\
\text { enhancer in B-cells } 2 \text { (p49/p100) }\end{array}$ & 1.54 & 1.03 & $0 \cdot 18$ & -1.02 & 0.16 & 0.218 \\
\hline 10280 & OPRS1 & Opioid receptor, sigma 1 & $-1 \cdot 28$ & -1.05 & 0.15 & -1.05 & 0.15 & 0.963 \\
\hline 5339 & PLEC1 & $\begin{array}{l}\text { Plectin 1, intermediate filament binding protein } \\
500 \mathrm{kDa}\end{array}$ & $-2 \cdot 14$ & $-1 \cdot 16$ & $0 \cdot 18$ & -1.08 & 0.21 & 0.206 \\
\hline 59339 & PLEKHA2 & $\begin{array}{l}\text { Pleckstrin homology domain containing, family A } \\
\text { (phosphoinositide binding specific) member } 2\end{array}$ & $6 \cdot 38$ & -1.08 & $0 \cdot 17$ & -1.05 & $0 \cdot 18$ & 0.668 \\
\hline 25939 & SAMHD1 & SAM domain and HD domain 1 & $5 \cdot 04$ & -1.06 & 0.14 & -1.03 & 0.19 & 0.468 \\
\hline 52729 & SERPINB9 & $\begin{array}{l}\text { Serpin peptidase inhibitor, clade } \\
\text { B (ovalbumin), member } 9\end{array}$ & $2 \cdot 45$ & 1.09 & 0.22 & -1.04 & 0.14 & 0.033 \\
\hline $9126^{\star \star}$ & SMC3 & Structural maintenance of chromosomes 3 & 4.64 & $-1 \cdot 10$ & 0.11 & -1.05 & 0.15 & 0.209 \\
\hline $6648 \|^{* *}$ & SOD2 & Superoxide dismutase 2 , mitochondrial & 3.49 & 1.01 & 0.31 & -1.05 & 0.18 & 0.473 \\
\hline 20442†‡ & ST3GAL1 & ST3 beta-galactoside alpha-2,3-sialyltransferase 1 & -1.9 & 0.98 & 0.17 & 0.97 & 0.18 & 0.914 \\
\hline 7091 & TLE4 & $\begin{array}{l}\text { Transducin-like enhancer of split } 4 \text { (E(sp1) } \\
\text { homologue, Drosophila) }\end{array}$ & $2 \cdot 08$ & -1.04 & $0 \cdot 15$ & -1.04 & 0.11 & 0.817 \\
\hline 7124 & TNF & Tumour necrosis factor (TNF superfamily, member 2) & $-1 \cdot 17$ & $-1 \cdot 16$ & 0.29 & -1.03 & 0.37 & 0.322 \\
\hline
\end{tabular}

Entrez ID, Entrez gene identifier.

${ }^{*}$ Fold changes are given as arithmetic mean and standard deviation (if $n>3$, microarray $n$ 3, placebo and quercetin $n$ 20). $P$ is given for paired Student's $t$ test comparing placebo $v$. quercetin treatment.

†Data from ninety-six-well TaqMan ${ }^{\circledR}$ quantitative real-time PCR experiments (as opposed to LDA data).

$\ddagger$ Part of $O$-glycan biosynthesis pathway.

$\S$ Part of adipocytokine signalling pathway.

\| Found in haematopoiesis and immune system biological pathways.

If Found in apoptosis-related biological pathway.

${ }^{\star \star}$ Found in nucleic acid metabolism biological pathway.

role in cell death and Alzheimer's disease), plectin 1 (PLEC1; multiple probe sets $\mathrm{FC}-2 \cdot 1$; may regulate changes in lymphocytes during extravasation), transducin-like enhancer of split $4(\mathrm{E}(\mathrm{sp} 1)$ homologue (TLE4; multiple probe sets FC $2 \cdot 1) ; P<0.05)$, IQ motif containing GTPase activating protein 1 (IQGAP1; FC 26.4), metastasis-associated lung adenocarcinoma transcript 1 (MALAT1; multiple probe sets FC 1.5-17.4), monoglyceride lipase (MGLL; FC - 1.6; may play a role in the inflammatory response), SAM domain and HD domain 1 (SAMHD1; multiple probe sets FC 1.7-5.0; $P=0.01-0.08$; may play a role in inflammatory processes), glutathione peroxidase $3\left(G P X 3 ; \mathrm{FC}-1.6 ; \mathrm{H}_{2} \mathrm{O}_{2}\right.$ catabolic process and response to lipid hydroperoxide), MAX gene associated (MGA; FC - 2.0) and pleckstrin homology domain containing, family A (phosphoinositide binding specific) member 2 (PLEKHA2; FC $6.4 ; P=0.02)$ and additionally TNF- $\alpha(T N F)$. Technical replication of microarray results by qRT-PCR was conducted for two of the three subjects (not enough total RNA was available from the third subject). Twenty-two transcripts were measured with TaqMan ${ }^{\circledR}$ Low Density Arrays and two (namely C1GALT1 and ST3GAL1) were measured with TaqMan ${ }^{\circledR}$ Assay in the conventional ninety-six-well format. Comparison of FC values, calculated from microarray and qRT-PCR data, showed a correlation for the majority of genes (Supplemental Fig. S6).

\section{Dose-dependent expression of selected genes}

Expression of twenty-four selected genes was measured by qRT-PCR in subjects receiving different doses of quercetin during a 2-week period (50, 100 and $150 \mathrm{mg} / \mathrm{d}$; study I). Three genes were positively correlated to dose-dependent expression changes after linear regression analysis, with $R^{2}$ values of 0.454 (IQGAP1), 0.711 (MALATI) and 0.720 $(C P T 1 B)$, as shown in Fig. 2. FC values of all genes and supplementation groups are given in Supplemental Table S7. 


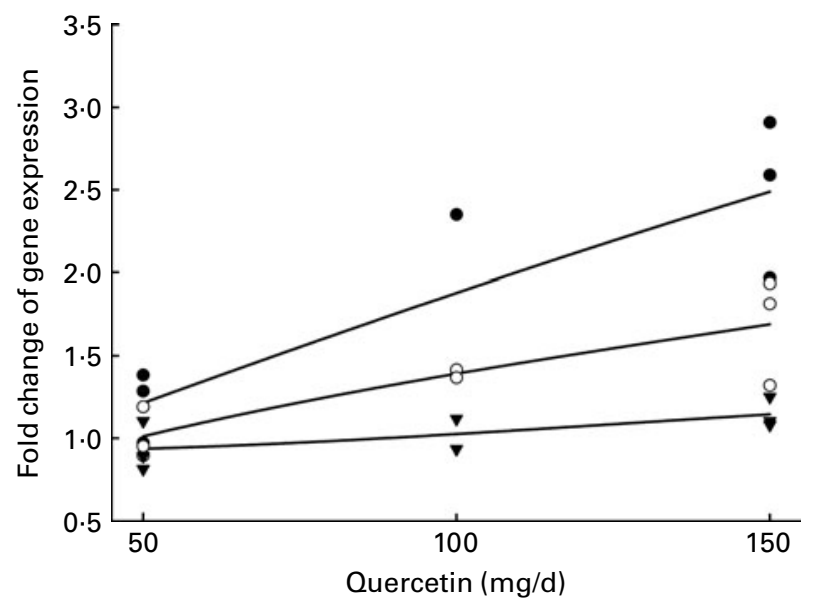

Fig. 2. Quercetin dose-dependent changes in gene expression. Fold change is given as change in gene expression after 2 weeks' supplementation with quercetin at $50 \mathrm{mg}(n 3), 100 \mathrm{mg}(n 2)$ or $150 \mathrm{mg}(n 3)$ per d compared with baseline expression, measured by quantitative real-time PCR. Fold changes of the three transcripts with the highest $R^{2}$ values are plotted against the quercetin dose and corresponding linear regression graphs are given (-). $R^{2}$ values are: $C P T 1 B$, carnitine palmitoyltransferase $1 \mathrm{~B}(\mathrm{O}) 0.720$; $M A L A T 1$, metastasis-associated lung adenocarcinoma transcript 1 (•) 0.711 ; IQGAP, IQ motif containing GTPase-activating protein $1(\boldsymbol{\nabla}) 0.454$.

\section{Expression of selected genes in subjects with} a CVD risk phenotype

Changes in expression of twenty-four genes identified by microarray analysis as differentially expressed in response to quercetin treatment in CD14-positive monocytes of healthy subjects (study I) were measured in a double-blind, placebocontrolled, cross-over study in subjects exhibiting a 'cardiovascular risk phenotype' (study II). As shown in Table 5, none of the selected genes showed similar changes in expression compared with results from study I and study II. Comparing gene expression changes between placebo and quercetin phases showed significant differences for only three genes: $G M 2 A$ (placebo phase FC 1.08 (SD 0.32) v. quercetin phase -1.08 (SD 0.16); $P=0.043), H D G F(\mathrm{FC}-1.15$ $(\mathrm{SD} 0.14) \quad v .-1.05(\mathrm{SD} 0.16) ; P=0.049)$ and SERPINB9 (1.09 (SD 0.22) v. -1.04 (SD 0.14); $P=0.033)$. Measurements of two $O$-glycan biosynthesis pathway genes (C1GALT1 and ST3GAL1) with ninety-six-well TaqMan ${ }^{\circledR}$ qRT-PCR (Table 5) showed no significant differences in expression between quercetin and placebo treatment for ST3GAL1. Expression of C1GALT1 was significantly higher after the placebo treatment compared with the quercetin treatment (FC 1.23 (SD 0.42) v. 1.02 (SD 0.22); $P=0.034)$. Comparison of $T N F$ mRNA and TNF- $\alpha$ protein FC showed no correlation (data not shown).

\section{Discussion}

We examined the effects of a quercetin supplementation on human CD14-positive monocyte gene expression by means of microarray analysis and evaluated the results in an independent, placebo-controlled study. Peripheral blood mononuclear cells (PBMC), consisting of lymphocytes and monocytes, are easily available and have frequently been used for analysis of gene expression profiles in human subjects. A number of studies have shown relatively low intra-individual variation compared with inter-individual differences in gene expression of PBMC ${ }^{(29-31)}$. But as Eady et al. ${ }^{(29)}$ and Whitney et al. ${ }^{(31)}$ observed, the composition of leucocyte subpopulations influences gene expression profiles in PBMC. More stable gene expression profiles can be obtained by using leucocyte subsets rapidly purified by positive selection, as shown by Lyons et $a l .^{(32)}$. We, therefore, used CD14-positive monocytes in our studies to minimise the influence of different leucocyte subpopulations on gene expression variability within and between individuals. Nevertheless, the processing time, from taking blood samples to extraction of RNA, has a profound influence on gene expression ${ }^{(28,31)}$. In the present study we aimed to minimise the storage time of blood samples at $4^{\circ} \mathrm{C}$ before further processing to less than $2 \mathrm{~h}$.

As has been discussed before, the effect of a dietary intervention on gene expression may be moderate but is nevertheless significant at the more complex levels of biological pathways and processes ${ }^{(16,33)}$. Our data analysis approach provided a high confidence in the gene expression being influenced mainly by quercetin supplementation instead of inter-individual variation. Second, differentially expressed genes were chosen on the basis of the magnitude of FC (at least \pm 1.5 ) and statistically significant difference in expression $(P \leq 0.01$ and $\mathrm{FC} \geq 1 \cdot 3)$. Based on these stringent criteria, functional enrichment analysis of the identified 'quercetin-regulated' genes resulted in a number of biological processes and pathways being significantly over-represented within this list. In accordance with previous observations we found biological processes of the immune system, apoptosis, nucleic acid metabolism and cancer-related pathways affected by quercetin supplementation ${ }^{(13,15-17)}$. On the other hand, a number of processes described previously being affected by quercetin were not significantly enriched in the present study including amino acid metabolism ${ }^{(17,20)}$, cell-cycle regulation $^{(13,15)}$ and lipid metabolism ${ }^{(14,16,20)}$. These differences may originate from the diverse experimental set up of the studies (including the small sample size in study I), utilising cell culture (CaCo-2, CO115, SH-SY5Y), rodent models and, within animal models, different tissues (lung, liver, colon, ileum, jejunum), which are not available in most human studies. Additionally, we identified a significant number of genes of the $O$-glycan biosynthesis pathway as differentially expressed, which has not been described before. Posttranslational $O$-glycosylation of selectins plays an important role in cell adhesion processes, especially capture and adhesion of leucocytes by endothelial cells during inflammation $^{(34,35)}$. While core 2 structures formed via GCNT1 seem to be important for leucocyte rolling ${ }^{(36)}$, ST3GAL1 competes with GCNT1 for the same substrate ${ }^{(35)}$. An increased formation of core 2 structures via GCNT1 and decreased substrate competition with ST3GAL1 would suggest no impact on leucocyte adhesion, which stands in contrast to possible anti-atherosclerotic effects of quercetin.

One biological pathway consistently found to be affected by quercetin is apoptosis. In cancer this is thought to contribute to the anticarcinogenic properties of quercetin ${ }^{(15)}$. Although cancer chemoprevention may not play a critical role in the studied cell type, cardiovascular health is another beneficial effect which has been reported for quercetin. But how could a pro-apoptotic action of quercetin benefit this? While 
enhanced apoptosis of macrophages in atherosclerotic plaques is thought to destabilise these structures $^{(37)}$, it has also been shown that enhanced apoptosis of circulating monocytes can reduce plaque development and so limit the progression of atherosclerosis ${ }^{(38,39)}$. The link between quercetin and antiatherosclerosis has been further highlighted by Kawai et al. showing that quercetin-3-glucuronide specifically accumulates in activated macrophages of atherosclerotic lesions ${ }^{(10)}$. Taken together, the results of previous studies and our microarray experiment suggest that quercetin may act via pro-apoptotic pathways on monocytes and possibly also activated macrophages and thus exert an anti-atherosclerotic effect.

Results obtained in microarray studies have often been verified only by replicated measurements of a few genes in the same samples or study populations with different methods $^{(15,16,33)}$. Here we used an independent study population to verify the effect of quercetin on the expression of selected genes identified in study I. Subjects of study II exhibited a CVD risk phenotype. The latent inflammatory state and combination of several risk factors present in these subjects may be more prone to a response after quercetin treatment, as Boots et al. have suggested ${ }^{(18)}$. However, we could not replicate the results found in our microarray experiment regarding the expression of twenty-four selected genes. The changes in gene expression after quercetin supplementation were significantly different from expression changes after placebo treatment only in four of twenty-four genes. The differences between initial microarray results (study I) and replication (study II) could be due to a number of factors. On the one hand, subjects in both studies were recruited based on different inclusion criteria. Although this was thought to result in a more distinct effect of quercetin on gene expression in study II, this might only be true for a different subset of genes. It is also possible that the severity of the CVD risk phenotype was already too pronounced to be affected by quercetin. Furthermore, the number of subjects examined by microarray analysis in study I was much smaller compared with study II. Also we only examined a small number of genes differentially expressed in the microarray experiment (twenty-four out of 788) and, although we feel we have used appropriate decision criteria for choosing these, the effect of quercetin on the expression of other genes might still prove reproducible. The considerable inter-individual variability in quercetin plasma concentrations complicates the interpretation of expression data. This variability was more evident in study I, probably due to the small number of subjects as can be seen in the high standard deviation. The overall variability of plasma quercetin concentrations is possibly based on inter-individual differences in bioavailability, additionally influencing the reproducibility of gene expression analyses.

In conclusion, we have shown with whole transcriptome analysis that quercetin affects gene expression of CD14positive monocytes in vivo, confirming previous reports that suggest pathways such as apoptosis are involved. We also found first evidence of quercetin possibly affecting $O$-glycosylation. Replication of these results in a separate study was, however, not successful, emphasising the importance of using stringent and most notably a placebo-controlled study design in order to identify gene expression regulated by flavonoids such as quercetin.

\section{Acknowledgements}

The present research was financially supported by the German Federal Ministry of Education and Research (BMBF), 'Functional Foods for Vascular Health - from Nutraceuticals to Personalised Diets' (grant no. FKZ 0313856A).

We thank D. Hallack and Y. Dignal for excellent technical assistance, and S. Schreiber and R. Häsler of the Institute of Clinical Molecular Biology at the University Hospital Schleswig-Holstein for their cooperation to use the microarray and LDA scanning facilities.

I. B. and F. D. designed the research. S. E. and M. J. M. designed the human intervention studies. I. B. performed the research and data analysis. S. E. performed the intervention studies. S. W. carried out the quercetin measurements. I. B. wrote the manuscript. F. D. and G. R. contributed to the discussion of results and final version of the manuscript. All authors reviewed the final manuscript.

The authors declare no conflicts of interest.

Supplemental figures and tables are available online only at http://journals.cambridge.org/action/displayJournal?jid=bjn

\section{References}

1. Hertog MG, Feskens EJ, Hollman PC, et al. (1993) Dietary antioxidant flavonoids and risk of coronary heart disease: the Zutphen Elderly Study. Lancet 342, 1007-1011.

2. Sampson L, Rimm E, Hollman PC, et al. (2002) Flavonol and flavone intakes in US health professionals. J Am Diet Assoc 102, 1414-1420.

3. Day AJ, Mellon F, Barron D, et al. (2001) Human metabolism of dietary flavonoids: identification of plasma metabolites of quercetin. Free Radic Res 35, 941-952.

4. Arts IC \& Hollman PC (2005) Polyphenols and disease risk in epidemiologic studies. Am J Clin Nutr 81, 317S-325S.

5. Williamson G \& Manach C (2005) Bioavailability and bioefficacy of polyphenols in humans. II. Review of 93 intervention studies. Am J Clin Nutr 81, 243S-255S.

6. Ames BN (1983) Dietary carcinogens and anticarcinogens. Oxygen radicals and degenerative diseases. Science 221, $1256-1264$.

7. Halliwell B (1993) The role of oxygen radicals in human disease, with particular reference to the vascular system. Haemostasis 23, Suppl. 1, 118-126.

8. Houstis N, Rosen ED \& Lander ES (2006) Reactive oxygen species have a causal role in multiple forms of insulin resistance. Nature 440, 944-948.

9. Formica JV \& Regelson W (1995) Review of the biology of quercetin and related bioflavonoids. Food Chem Toxicol 33, $1061-1080$

10. Kawai Y, Nishikawa T, Shiba Y, et al. (2008) Macrophage as a target of quercetin glucuronides in human atherosclerotic arteries: implication in the anti-atherosclerotic mechanism of dietary flavonoids. J Biol Chem 283, 9424-9434.

11. Lodi F, Jimenez R, Moreno L, et al. (2009) Glucuronidated and sulfated metabolites of the flavonoid quercetin prevent endothelial dysfunction but lack direct vasorelaxant effects in rat aorta. Atherosclerosis 204, 34-39.

12. Boesch-Saadatmandi C, Pospissil RT, Graeser AC, et al. (2009) Effect of quercetin on paraoxonase 2 levels in RAW264.7 macrophages and in human monocytes - role of quercetin metabolism. Int J Mol Sci 10, 4168-4177.

13. Murtaza I, Marra G, Schlapbach R, et al. (2006) A preliminary investigation demonstrating the effect of quercetin on the expression of genes related to cell-cycle arrest, apoptosis and 
xenobiotic metabolism in human CO115 colon-adenocarcinoma cells using DNA microarray. Biotechnol Appl Biochem 45, $29-36$.

14. Soundararajan R, Wishart AD, Rupasinghe HP, et al. (2008) Quercetin 3-glucoside protects neuroblastoma (SH-SY5Y) cells in vitro against oxidative damage by inducing sterol regulatory element-binding protein-2-mediated cholesterol biosynthesis. J Biol Chem 283, 2231-2245.

15. van Erk MJ, Roepman P, van der Lende TR, et al. (2005) Integrated assessment by multiple gene expression analysis of quercetin bioactivity on anticancer-related mechanisms in colon cancer cells in vitro. Eur J Nutr 44, 143-156.

16. de Boer VC, van Schothorst EM, Dihal AA, et al. (2006) Chronic quercetin exposure affects fatty acid catabolism in rat lung. Cell Mol Life Sci 63, 2847-2858.

17. Dihal AA, van der Woude H, Hendriksen PJ, et al. (2008) Transcriptome and proteome profiling of colon mucosa from quercetin fed F344 rats point to tumor preventive mechanisms, increased mitochondrial fatty acid degradation and decreased glycolysis. Proteomics 8, 45-61.

18. Boots AW, Wilms LC, Swennen EL, et al. (2008) In vitro and $e x$ vivo anti-inflammatory activity of quercetin in healthy volunteers. Nutrition 24, 703-710.

19. de Pascual-Teresa S, Johnston KL, DuPont MS, et al. (2004) Quercetin metabolites downregulate cyclooxygenase-2 transcription in human lymphocytes ex vivo but not in vivo. J Nutr 134, $552-557$.

20. Mutch DM, Crespy V, Clough J, et al. (2006) Hepatic cytochrome P-450 reductase-null mice show reduced transcriptional response to quercetin and reveal physiological homeostasis between jejunum and liver. Am J Physiol Gastrointest Liver Physiol 291, G63-G72.

21. Egert S, Wolffram S, Bosy-Westphal A, et al. (2008) Daily quercetin supplementation dose-dependently increases plasma quercetin concentrations in healthy humans. J Nutr 138, 1615-1621.

22. Egert S, Bosy-Westphal A, Seiberl J, et al. (2009) Quercetin reduces systolic blood pressure and plasma oxidised low-density lipoprotein concentrations in overweight subjects with a high-cardiovascular disease risk phenotype: a double-blinded, placebo-controlled cross-over study. Br J Nutr 102, 1065-1074.

23. Klapper M, Dopner M, Vock C, et al. (2006) Expression analysis of genes involved in fat assimilation in human monocytes. IUBMB Life 58, 435-440.

24. Dennis G Jr, Sherman BT, Hosack DA, et al. (2003) DAVID: Database for Annotation, Visualization, and Integrated Discovery. Genome Biol 4, P3.
25. Brazma A, Hingamp P, Quackenbush J, et al. (2001) Minimum information about a microarray experiment (MIAME) - toward standards for microarray data. Nat Genet 29, 365-371.

26. Schmelzer C, Lorenz G, Rimbach G, et al. (2007) Influence of coenzyme $\mathrm{Q}_{10}$ on release of pro-inflammatory chemokines in the human monocytic cell line THP-1. Biofactors 31, $211-217$

27. Tsuchiya S, Yamabe M, Yamaguchi Y, et al. (1980) Establishment and characterization of a human acute monocytic leukemia cell line (THP-1). Int J Cancer 26, 171-176.

28. Livak KJ \& Schmittgen TD (2001) Analysis of relative gene expression data using real-time quantitative PCR and the $2^{-\Delta \Delta \mathrm{C}} \mathrm{T}$ method. Methods 25, 402-408.

29. Eady JJ, Wortley GM, Wormstone YM, et al. (2005) Variation in gene expression profiles of peripheral blood mononuclear cells from healthy volunteers. Physiol Genomics 22, 402-411.

30. Radich JP, Mao M, Stepaniants S, et al. (2004) Individualspecific variation of gene expression in peripheral blood leukocytes. Genomics 83, 980-988.

31. Whitney AR, Diehn M, Popper SJ, et al. (2003) Individuality and variation in gene expression patterns in human blood. Proc Natl Acad Sci U S A 100, 1896-1901.

32. Lyons PA, Koukoulaki M, Hatton A, et al. (2007) Microarray analysis of human leucocyte subsets: the advantages of positive selection and rapid purification. BMC Genomics 8, 64.

33. Pagmantidis V, Meplan C, van Schothorst EM, et al. (2008) Supplementation of healthy volunteers with nutritionally relevant amounts of selenium increases the expression of lymphocyte protein biosynthesis genes. Am J Clin Nutr 87, 181-189.

34. McEver RP (2002) Selectins: lectins that initiate cell adhesion under flow. Curr Opin Cell Biol 14, 581-586.

35. Sperandio M (2006) Selectins and glycosyltransferases in leukocyte rolling in vivo. FEBS J 273, 4377-4389.

36. Sperandio M, Thatte A, Foy D, et al. (2001) Severe impairment of leukocyte rolling in venules of core 2 glucosaminyltransferase-deficient mice. Blood 97, 3812-3819.

37. Babaev VR, Chew JD, Ding L, et al. (2008) Macrophage EP4 deficiency increases apoptosis and suppresses early atherosclerosis. Cell Metab 8, 492-501.

38. Ermak N, Lacour B, Drueke TB, et al. (2008) Role of reactive oxygen species and Bax in oxidized low density lipoprotein-induced apoptosis of human monocytes. Atherosclerosis 200, 247-256.

39. Stoneman V, Braganza D, Figg N, et al. (2007) Monocyte/ macrophage suppression in CD11b diphtheria toxin receptor transgenic mice differentially affects atherogenesis and established plaques. Circ Res 100, 884-893. 